\title{
Constraining portals with displaced Higgs decay searches at the LHC
}

\author{
Jackson D. Clarke \\ ARC Centre of Excellence for Particle Physics at the Terascale, \\ School of Physics, University of Melbourne, 3010, Australia \\ E-mail: j.clarke5@pgrad.unimelb.edu.au
}

ABSTRACT: It is very easy to write down models in which long-lived particles decaying to standard model states are pair-produced via Higgs decays, resulting in the signature of approximately back-to-back pairs of displaced narrow hadronic jets and/or lepton jets at the LHC. The LHC collaborations have already searched for such signatures with no observed excess. This paper describes a Monte Carlo method to reinterpret the searches. The method relies on (ideally multidimensional) efficiency tables, thus we implore collaborations to include them in any future work. Exclusion regions in mixing-mass parameter space are presented which constrain portal models.

KEYwords: Phenomenological Models, Hadronic Colliders

ARXIV EPRINT: 1505.00063 


\section{Contents}

1 Introduction 1

2 Models 2

3 Method and results $\quad 4$

3.1 Displaced hadronic jets 4

$\begin{array}{lll}3.2 & \text { Displaced lepton jets } & 7\end{array}$

4 Portal limits $\quad 10$

5 Conclusion $\quad 12$

\section{Introduction}

One of the primary goals of the LHC programme is to study the properties of the recently discovered $\approx 125 \mathrm{GeV}$ state, $h$, in great detail $[1,2]$. So far the state is consistent with a standard model (SM) Higgs. Still, plenty of room remains for new physics, particularly in its decays; $\operatorname{Br}(h \rightarrow$ unobserved $) \lesssim 20 \%$ is still allowed for an otherwise SM Higgs [3, 4]. It is therefore sensible to consider spectacular Higgs decay channels for which searches might have high sensitivity. Of interest in this paper is one such channel: pair production of long-lived states which decay on detector length scales [5].

Long-lived neutral states decaying to SM particles arise naturally in models with approximate symmetries. There are four mechanisms often encountered in the literature: an approximate enhanced Poincaré symmetry [6], which if exact would completely decouple a hidden sector; an approximate discrete symmetry, which if exact would produce a stable lightest particle; a low-energy accidental symmetry, with decays only proceeding via a heavy off-shell mediator, and; small mass-splittings. The first mechanism is associated with the usual portal models.

Testable models in which a SM-like Higgs decays to pairs of these long-lived states are easy to build. The generic signature is an approximately back-to-back pair of displaced narrow hadronic jets and/or lepton jets [7] (although this is certainly not the only possibility), for which null searches have already been performed by the LHC collaborations [8-18]. The peculiarity of the signature presents two complementary challenges: how do collaborations present their results in the most model-independent way possible? and how do phenomenologists reinterpret the results in the context of their own models? This paper describes a simple Monte Carlo method which has implications for both. The short message is the following: if the relevant efficiency tables are published, then phenomenologists need only take Monte Carlo events and fold in these efficiencies to reinterpret searches. No displaced decays need be simulated since decay probabilities are easily calculated. 
The outline of this paper is as follows. In section 2 we write down some simple models of interest with an emphasis on portal models. In section 3 we describe a method for recasting displaced searches by way of example, reinterpreting two ATLAS searches. Exclusion regions in mixing-mass parameter space are presented which apply to portal models; they are explicitly applied to the Higgs and vector portals in section 4. Section 5 concludes.

\section{Models}

In this section we write down some models of interest which should serve to illustrate (a) how easy it is to write down a natural model with long-lived states, (b) how these states can inherit very different couplings to SM particles, and (c) how an extended model can introduce non-trivial final states. Thereupon it should be clear that displaced searches would benefit from a model-independent approach.

1. SM plus real singlet scalar: with an extra real singlet scalar field $S$, the SM potential becomes

$$
V=-\mu^{2} \phi^{\dagger} \phi+\lambda \phi^{\dagger} \phi \phi^{\dagger} \phi+\zeta \phi^{\dagger} \phi S^{2}+\xi \phi^{\dagger} \phi S-\mu_{s}^{2} S^{2}+\lambda_{s} S^{4}+\alpha S+\beta S^{3}
$$

where $\phi$ is the Higgs field. The limits $\alpha, \beta, \xi \rightarrow 0$ and $\zeta, \xi \rightarrow 0$ are technically natural.

Once $\phi$ develops a non-zero vacuum expectation value (VEV), the cubic term $\xi \phi^{\dagger} \phi S$ induces mixing between the SM Higgs doublet state $\phi_{0}^{\prime}$ and $S$ to produce mass eigenstates

$$
\left(\begin{array}{l}
h \\
s
\end{array}\right)=\left(\begin{array}{cc}
\cos \rho & -\sin \rho \\
\sin \rho & \cos \rho
\end{array}\right)\left(\begin{array}{c}
\phi_{0}^{\prime} \\
S^{\prime}
\end{array}\right)
$$

where $\rho \approx \sin \rho \ll 1$ is a mixing angle and $h$ is to be identified with the observed SMlike Higgs. Then $s$ decays to SM particles through mixing, with a rate $\propto \rho^{2}$, leading to displaced decays when $\rho$ is very small.

If $\mu_{s}^{2}<0$, then to first order in $\alpha, \beta, \xi$, and $\zeta$, the mixing $\rho$ and the effective hss coupling $\kappa$ are

$$
\tan \rho=\frac{\xi v}{\left|m_{h}^{2}-m_{s}^{2}\right|}, \quad \kappa=\zeta v
$$

where $v \approx 246 \mathrm{GeV}$.

Terms odd in $S$ can be forbidden by demanding some symmetry, for example $Z_{2}(S \rightarrow$ $-S$ ) or classical scale invariance. Then $s=S$ is stable and a dark matter candidate [19] unless $\mu_{s}^{2}>0$ and an effective cubic term is generated by $S$ acquiring a non-zero VEV $\langle S\rangle=v_{s}$. In this case, at tree-level and to leading order in $\zeta$,

$$
\tan \rho=\frac{\zeta v v_{s}}{\left|m_{h}^{2}-2 m_{s}^{2}\right|}=\zeta v \frac{m_{s}}{\sqrt{\lambda_{s}}\left|m_{h}^{2}-2 m_{s}^{2}\right|}, \quad \kappa=\zeta v \frac{m_{h}^{2}+4 m_{s}^{2}}{m_{h}^{2}-2 m_{s}^{2}} .
$$

This is the usual Higgs portal model [20, 21]. 
The phenomenology of $\mathrm{GeV}$-scale scalars is detailed in ref. [22]. We note the significant QCD uncertainties in the widths for $2 m_{\pi}<m_{s} \lesssim 4 \mathrm{GeV}$. The decay width of the Higgs to light scalars is

$$
\Gamma(h \rightarrow s s)=\frac{\kappa^{2}}{32 \pi m_{h}} \sqrt{1-\frac{4 m_{s}^{2}}{m_{h}^{2}}} .
$$

Thus for fixed $\operatorname{Br}(h \rightarrow s s)$ and $m_{s}, \kappa$ is fully determined. ${ }^{1}$

2. Two-Higgs-doublet model (2HDM) plus singlet scalar: after symmetry breaking in the CP-conserving $2 \mathrm{HDM}$, one ends up with five Higgs fields, of which the neutrals are written $h, H, A$ (see ref. [23] for details). In the decoupling limit, $\alpha \rightarrow \pi / 2-\beta, h$ can be associated with the SM-like Higgs. To avoid tree-level flavour-changing neutral currents, a symmetry is usually demanded to ensure that each of $\left(u_{R}^{i}, d_{R}^{i}, e_{R}^{i}\right)$ couple to only one of the Higgs doublets [24, 25]. The four arrangements, known as Type I, Type II, Lepton-specific and Flipped, qualitatively alter the couplings of, in particular, $H$ and $A$ to the SM fermions (see table 2 of ref. [23]).

In the $2 \mathrm{HDM}$ with an extra singlet, if the singlet develops a non-zero VEV then its real (imaginary) part can mix with $h / H(A)$ to form a mass eigenstate $s(a)$ which could be very light. Depending on their admixture of 2HDM Higgs', the fermion coupling type, and the values of $\tan \beta$ and $\alpha, s$ and $a$ could couple dominantly to up-types, down-types, or leptons, as demonstrated in figures 7-10 of ref. [26]. Additionally, if these states are mostly singlet then their lifetimes can be long.

3. Dark photon: a dark photon $\gamma_{d}$ can arise if an extra $\mathrm{U}(1)_{d}$ gauge group is appended to the SM [27]. The SM Lagrangian can be extended by

$$
\mathcal{L}_{D}=-\frac{1}{4} F_{\mu \nu}^{\prime} F^{\prime \mu \nu}-\frac{\epsilon}{2 \cos \theta_{W}} F_{\mu \nu}^{\prime} B^{\mu \nu},
$$

where $F^{\prime \mu \nu}\left(B^{\mu \nu}\right)$ is the $\mathrm{U}(1)_{d}$ (hypercharge) field strength, $\theta_{W}$ is the Weinberg angle, and $\epsilon$ is the kinetic mixing parameter. If the $\gamma_{d}$ is massive, and $m_{\gamma_{d}} \ll m_{Z}$, the couplings of $\gamma_{d}$ to SM particles are photon-like and $\propto \epsilon^{2}{ }^{2}$ The lifetime of such a $\gamma_{d}$ is $\propto \epsilon^{-2}$, and if $\epsilon$ is small enough the $\gamma_{d}$ can be long-lived.

A mass term for $\gamma_{d}$ can be generated through a Higgs mechanism [30-32]. The simplest way is to introduce a complex scalar field with a potential

$$
V=-\mu_{s}^{2}|S|^{2}+\lambda_{s}|S|^{4}+\zeta|\phi|^{2}|S|^{2},
$$

such that $S$ acquires a VEV, leaving a massive state $s$ and giving mass $m_{\gamma_{d}} \approx g_{d} v_{s}$ to the dark photon, where $g_{d}$ is the dark gauge coupling. ${ }^{3}$ In this case, the widths of the

\footnotetext{
${ }^{1}$ Note that the requirement $\lambda_{s}<4 \pi$ implies $\rho^{2} \gtrsim 2 \times 10^{-9}\left(\frac{m_{s}}{\mathrm{GeV}}\right)^{2} \frac{B r(h \rightarrow s s)}{0.1}$ in the case of eq. (2.4). There is no such implication in the eq. (2.3) case.

${ }^{2}$ In a general model this need not be the case, e.g. it is possible for the extra $\mathrm{U}(1)_{d}$ field to mass mix with the SM $Z$ boson independently of $\epsilon[28,29]$.

${ }^{3}$ This scenario is natural in the limit $\epsilon, \zeta \rightarrow 0$ and $\epsilon \rightarrow 0$. Note that an effective $\zeta$ is generated at the level $\sim g_{d} g \epsilon^{2} \times$ (loop factor) by the kinetic mixing term.
} 
SM-like Higgs to $\gamma_{d} \gamma_{d}$ and $s s$ are $\propto \zeta^{2}$, and it is easy to obtain branchings of $\mathcal{O}(10 \%)$ when $2 m_{s}$ and/or $2 m_{\gamma_{d}}$ are less than $m_{h}[26,33,34]$. If $2 m_{\gamma_{d}}<m_{s}$ then $s$ decays promptly via $s \rightarrow \gamma_{d} \gamma_{d}$. Otherwise, it turns out, for $m_{s}, m_{\gamma_{d}} \sim \mathrm{GeV}$ it is possible that $\epsilon$ and $\zeta$ take values which result in $\mathcal{O}(10 \%)$ Higgs branchings and either $\gamma_{d}$, or $s$, or both long-lived (see refs. $[35,36]$ for some discussion).

4. Other models: here we comment on other contexts in which SM-like Higgs decays to long-lived states have arisen in the literature. We note that many of these models also predict other long-lived state production mechanisms which can be considered independently.

Long-lived right-handed neutrinos can be pair produced and decay to fermion trilinears [37-40]. In R-parity violating supersymmetry, the would-be neutralino dark matter candidate can be long-lived, decaying to fermion bi- or trilinears (see e.g. [41-45]). The neutralinos may be pair produced directly or in a cascade if the spectrum below $m_{h}$ is sufficiently complex. Models of WIMP baryogenesis also generically predict long-lived particles which decay via fermion trilinears [46]. In more complex models with a light hidden sector, such as hidden valley models, cascades are common [7, 47-49], producing large multiplicity final states often associated with missing energy. In a hidden sector with a confining gauge group one expects bound states [50]. If the confinement scale is $\sim m_{h}$ then the SM-like Higgs could decay to hidden hadron pairs or hidden glueballs which then decay back to SM particles via a heavy mediator (or the Higgs portal) on collider length scales [5, 51]. Such phenomenology is typical of recent "neutral naturalness" models [52-54]. If the confinement scale is $\ll m_{h}$ then one expects hidden jets, which could result in a very large multiplicity of displaced vertices along with missing energy [55-57].

\section{Method and results}

In the following section we describe a Monte Carlo method for recasting displaced searches by way of example. The important point to be made is that phenomenologists cannot reliably simulate the detector response to displaced decays, and are therefore reliant upon efficiency information provided by the collaborations. ${ }^{4}$ The recast examples will serve to highlight which efficiency information is of most interest.

\subsection{Displaced hadronic jets}

The ATLAS Collaboration has presented a search for the displaced hadronic decays of pairproduced long-lived neutral particles in $20.3 \mathrm{fb}^{-1}$ of data collected at $\sqrt{s}=8 \mathrm{TeV}[9,10]$. They considered pair production via the parton process $g g \rightarrow \Phi \rightarrow \pi_{v} \pi_{v}$, where $\Phi$ is a scalar particle and $\pi_{v}$ is a hidden valley pseudoscalar. The decay of $\pi_{v}$ is dominated by $b \bar{b}$ for $m_{\pi_{v}} \gtrsim 10 \mathrm{GeV}$ (the $c \bar{c}$ and $\tau \tau$ decays are subdominant, see their table 1 ). No excess was observed, and limits were placed on the branching fraction of $\Phi$ as a function of $m_{\pi_{v}}$ lifetime. Presently we describe a method to reproduce the result.

\footnotetext{
${ }^{4}$ This point was also made (and a similar recast method was used) in ref. [46].
} 
Validation samples of $g g \rightarrow h \rightarrow s s \rightarrow(b \bar{b})(b \bar{b})$ events in $\sqrt{s}=8 \mathrm{TeV} p p$ collisions were generated using Pyтнia $8.180[58,59]$ with the default tune. We took $m_{h}=126 \mathrm{GeV}$ and $m_{s}=10,25,40 \mathrm{GeV}$ to match the ATLAS benchmarks. ${ }^{5}$

The cuts used in the ATLAS analysis are listed in the auxiliary table 6 of ref. [10]. We recreate them as follows. The pair produced long-lived particles are required to have ${ }^{6}$

$$
E_{T}\left(s_{1}\right)>60 \mathrm{GeV}, \quad E_{T}\left(s_{2}\right)>40 \mathrm{GeV},
$$

where $E_{T} \equiv E \sin \theta$ is a proxy for the measured transverse energy of the resulting jet, and the $s$ subscript indicates $p_{T}$-ordering. This is a fine approximation except for the (nonhadronic) $s \rightarrow \tau \tau$ decays with $\sim 10 \%$ branching. We also demand $\Delta R\left(s_{1}, s_{2}\right)>0.4$ to ensure well-separated jets; this makes very little impact on the benchmarks considered by ATLAS, but will matter as $m_{s} \rightarrow m_{h} / 2$. None of the relevant remaining cuts, such as on isolation and on electromagnetic fraction, nor the trigger efficiency can be replicated since no public tool exists to simulate the detector response to displaced decays. ${ }^{7}$ Therefore the remainder of the analysis necessarily involves the folding in of efficiencies provided by ATLAS.

The CalRatio trigger $[62,63]$ was used to search for $\pi_{v}$ decays at or beyond the edge of the electromagnetic calorimeter. This trigger selects narrow jets with $E_{T} \gtrsim 35 \mathrm{GeV}$, $\log _{10}\left(E_{H} / E_{E M}\right)>1.2$, and a lack of tracks in the inner detector. The trigger efficiency is given as a function of radial (longitudinal) decay position for decays in the barrel (endcap) region corresponding to the pseudorapidity region $|\eta|<1.5(1.5<|\eta|<2.5)$ in figure 1 of ref. [9]. Based on these plots we take the trigger+reconstruction efficiency of the trigger jet to be non-zero and constant only between 2.0 to $3.5 \mathrm{~m}$ in the barrel and 4.0 to $5.5 \mathrm{~m}$ in the endcap, with a respective ratio of $0.20 / 0.06$. The reconstruction efficiency for the nontrigger jet is not given, but we take it similarly. By construction, the following quantity is then proportional to the trigger/reconstruction probability for a given $s$ of lifetime $c \tau$ :

$$
\hat{\varepsilon}(s, c \tau)=\left\{\begin{array}{l}
0.20 P(s, c \tau) \text { if in barrel, } \\
0.06 P(s, c \tau) \text { if in endcap, }
\end{array}\right.
$$

where $P$ is the probability that a state $x$ decays between $L_{\min }$ and $L_{\max }$,

$$
P(x, c \tau)=-\exp \left(-\frac{L_{\max }}{\gamma \beta c \tau}\right)+\exp \left(-\frac{L_{\min }}{\gamma \beta c \tau}\right),
$$

with $\gamma$ and $\beta$ the relativistic parameters for $x$. The timing of the $s$ decay is required to satisfy $\Delta t<5$ ns with respect to a $\beta=1$ particle. This corresponds to requiring an absolute decay distance

$$
L_{\text {abs }}<\frac{\beta}{1-\beta} 1.5 \mathrm{~m} \equiv L_{\text {abs }}^{\max } .
$$

\footnotetext{
${ }^{5}$ The accuracy of the Monte Carlo for an $s$ of mass $m_{s}=10 \mathrm{GeV}$ decaying directly to $b \bar{b}$ is questionable, nevertheless it is possible to force PYтнIA to do the decay, and it appears that this is what was done in the ATLAS analysis.

${ }^{6}$ Selections were made using the MADANALYsis5 v1.1.10beta SAMPLEANALYZER framework [60].

${ }^{7}$ Though some attempts have been made [49, 61].
} 
Thus after the aforementioned selection cuts, each event is weighted by a factor

$$
W(c \tau)=\hat{\varepsilon}\left(s_{1}, c \tau\right) \hat{\varepsilon}\left(s_{2}, c \tau\right),
$$

where we take

$$
\left(L_{\mathrm{min}}, L_{\mathrm{max}}\right)= \begin{cases}\left(\min \left(\frac{2.0 \mathrm{~m}}{\sin \theta}, L_{\mathrm{abs}}^{\mathrm{max}}\right), \min \left(\frac{3.5 \mathrm{~m}}{\sin \theta}, L_{\mathrm{abs}}^{\max }\right)\right) & \text { if in barrel, } \\ \left(\min \left(\frac{4.2 \mathrm{~m}}{\cos \theta}, L_{\mathrm{abs}}^{\max }\right), \min \left(\frac{5.2 \mathrm{~m}}{\cos \theta}, L_{\mathrm{abs}}^{\max }\right)\right) & \text { if in endcap, }\end{cases}
$$

for each $s$, where $\theta$ is the polar angle from the beam line. After this, the remainder of the cuts used in the ATLAS analysis should be largely independent of $c \tau$. As such, penultimately, we rescale the events (with a common number for all $c \tau$ and $m_{s}$ ) to fit ATLAS results; the factor turns out to be $\approx 6 \times 19.0 \mathrm{pb} \times 20.3 \mathrm{fb}^{-1} / N_{\text {sim }}$, where $N_{\text {sim }}$ is the number of simulated events. We ignore for simplicity the additional $m_{s}$-dependent $\lesssim 10 \%$ effect related to sub-dominant but non-zero $s \rightarrow \tau \tau$ branching.

After these requirements we find good agreement for the $m_{s}=10,25 \mathrm{GeV}$ samples, but we overpredict for $m_{s}=40 \mathrm{GeV}$. This is because as $m_{s}$ approaches $E_{s}$ (eq. (3.1)), the $s$ decay products spread out and the narrow jet trigger efficiency decreases. To properly take this into account we require information on how the efficiency depends on the $b \bar{b}$ opening angle, or equivalently, in the limit $E_{s}^{2} \gg m_{b}^{2}$, the boost. In the absence of such information, and in an attempt to capture the physics involved, we demand the following (admittedly crude) bound on the opening angle of the $b \bar{b}$ pair from the leading $s:^{8}$

$$
\Delta R(b, \bar{b})<1.5 \text {. }
$$

This cut has been tuned so that our results for $m_{s}=40 \mathrm{GeV}$ best agree with those of ATLAS. Note that the spatial separation of the $b \bar{b}$ pair as seen by the hadronic calorimeter is smaller than such a large $\Delta R$ would normally suggest, since the pair appears late.

In figure 1 (a) we compare the number of events predicted by our analysis to those of ATLAS assuming 100\% Higgs branching. Despite the apparent crudeness of some of our assumptions, we observe good agreement. The 95\% CL limit of 20 events (inferred from the ATLAS plots) can be used to obtain a limit on the Higgs exotic branching fraction as a function of $c \tau$. To obtain the exclusion for alternative masses, PyTHIA signal samples $g g \rightarrow$ $h \rightarrow s s \rightarrow(b \bar{b})(b \bar{b})$ of varying $m_{s}$ were fed through our analysis. In figure 1(b) we present our results as limits on $\operatorname{Br}(h \rightarrow s s) \times B r(s \rightarrow \text { hadronic })^{2}$ as a function of $m_{s}$ and $c \tau$. This is also a good approximation for the limit on $\operatorname{Br}\left(h \rightarrow \gamma_{d} \gamma_{d}\right) \times B r\left(\gamma_{d} \rightarrow\right.$ hadronic $^{2}$. Given the good match to ATLAS, we are confident that our results for $10 \mathrm{GeV}<m_{s}<40 \mathrm{GeV}$ are reliable, and for $m_{s}>40 \mathrm{GeV}$ are at least sensible. In section 4 , figure 1(b) is reinterpreted to bound mixing-mass parameter space for the Higgs and vector portals.

We were fortunate in this analysis because the topology of interest was essentially already considered by ATLAS for three benchmark values of $m_{s}$. This allowed us to

\footnotetext{
${ }^{8}$ Another option is to reject leading $s$ with boost lower than $\gamma_{c u t}$, forbidding particles which have the potential to produce opening angles $\approx \arccos \left[1-2 / \gamma_{\text {cut }}^{2}+8 m_{x}^{2}\left(\gamma_{c u t}^{2}-1\right) /\left(m_{s}^{2} \gamma_{c u t}^{4}\right)\right]$, where $m_{x}$ is the decay product mass.
} 


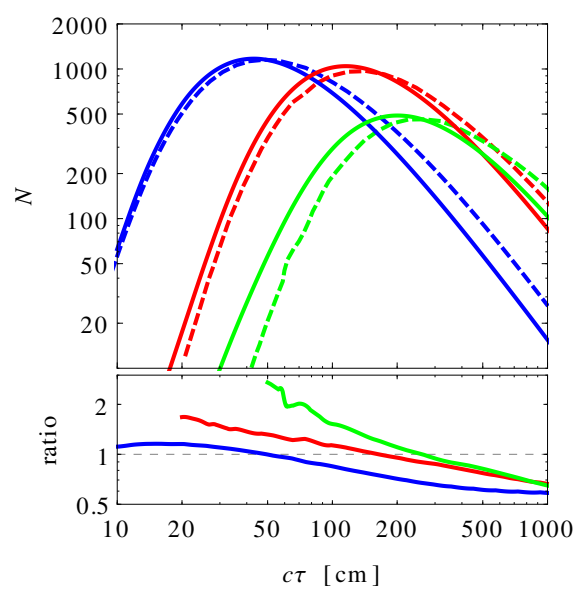

(a)

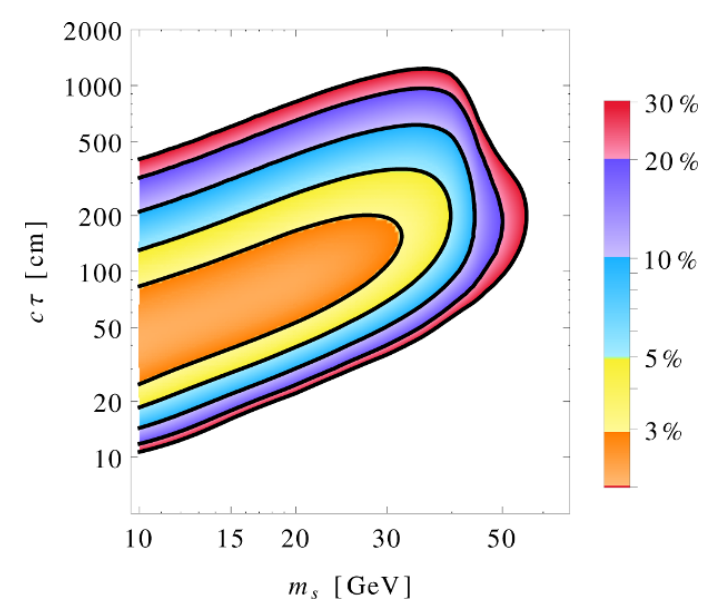

(b)

Figure 1. (a) Predicted number of events for the displaced hadronic jet analysis assuming $100 \%$ Higgs branching. Upper: the results from ATLAS (dashed) and our results (solid) for $m_{s}=$ $10,25,40 \mathrm{GeV}$ beginning left-to-right. Lower: the ratio of our results to those of ATLAS. (b) Excluded parameter region for $s$ assuming fixed $\mathrm{Br}(h \rightarrow s s) \times \mathrm{Br}\left(s \rightarrow\right.$ hadronic $^{2}$; the contours mark branchings of $30 \%, 20 \%, 10 \%, 5 \%, 3 \%$.

demonstrate the not obvious fact that much of the $c \tau$ dependence is taken into account simply by reweighting events with easily calculated decay probabilities (eq. (3.3)). In the region where $\gamma_{s} \sim 1$ we saw that there was an additional effect that had to be considered, related to the boost-dependence of the trigger/reconstruction efficiency. This could have been anticipated, since any momentum dependence was already integrated out of the efficiency plots provided by ATLAS. For this reason our analysis as it stands cannot be reliably reapplied to another model since the overall efficiency will scale non-trivially with the (correlated) $p_{T}$ distributions of the two long-lived particles. However it should serve as a conservative estimate for models with more boosted (on average) long-lived pairs. In the next analysis the $p_{T}$ dependence of the efficiencies is provided and taken into account.

\subsection{Displaced lepton jets}

In ref. [13], the ATLAS Collaboration presented the search for a SM-like Higgs decaying to a long-lived pair of $\mathcal{O}(100 \mathrm{MeV})$ dark photons in $20.3 \mathrm{fb}^{-1}$ of data collected at $\sqrt{s}=8 \mathrm{TeV}$. The benchmark process considered was

$$
g g \rightarrow h \rightarrow f_{d_{1}} \bar{f}_{d_{1}} \rightarrow\left(f_{d_{2}} \gamma_{d}\right)\left(\bar{f}_{d_{2}} \gamma_{d}\right)
$$

where the $f_{d_{i}}$ are hidden fermions and $\gamma_{d}$ is the long-lived dark photon, inspired by Falkowski-Ruderman-Volansky-Zupan (FRVZ) models [48, 49]. Each dark photon decays at or beyond the outer edge of the electromagnetic calorimeter to either $\mu \mu$ or $e e / \pi \pi$, resulting in a muon jet (Type 0) or a narrow jet (Type 2) respectively. ${ }^{9}$ No excess over the SM expectation was observed and limits were placed on the exotic branching fraction of the

\footnotetext{
${ }^{9}$ Note that electrons in the hadronic calorimeter will resemble a narrow hadronic jet.
} 
Higgs as a function of $\gamma_{d}$ lifetime. These limits are clearly model-dependent, and it is not clear how the phenomenologist might translate them. We describe a simple method below.

A validation sample of FRVZ events was generated in PyтніA $8.180[58,59]$ by changing the properties of in-built particles. We took $\left(m_{h}, m_{f_{d_{1}}}, m_{f_{d_{2}}}, m_{\gamma_{d}}\right)=(125,5,2,0.4) \mathrm{GeV}$ and $\operatorname{Br}\left(\gamma_{d} \rightarrow\right.$ Type 0$)=0.45$ to match the ATLAS benchmark.

The selection criteria (cuts) for the ATLAS analysis are detailed in table 1 of ref. [13]. We recreate the analysis as follows. The dark photons are required to satisfy

$$
\left|\eta\left(\gamma_{d}\right)\right|<2.5, \quad\left|\Delta \phi\left(\gamma_{d_{1}}, \gamma_{d_{2}}\right)\right|>1
$$

as a proxy for the jet acceptance. The remainder of the analysis necessarily involves the folding in of efficiencies. After each $\gamma_{d}$ decays, the final states are of Type 0-0, 0-2, or 2-2 in the obvious way. After selection, each event is weighted by a factor

$$
W(c \tau)=\frac{19.2 \mathrm{pb} \times 20.3 \mathrm{fb}^{-1}}{N_{\text {sim }}} P_{\text {reco }}\left(\gamma_{d_{1}}, c \tau\right) P_{\text {reco }}\left(\gamma_{d_{2}}, c \tau\right) \varepsilon_{\text {trig }}\left(\gamma_{d_{1}}, \gamma_{d_{2}}\right),
$$

where $P_{\text {reco }}\left(\gamma_{d}, c \tau\right)$ is the reconstruction probability ${ }^{10}$ for a $\gamma_{d}$ of lifetime $c \tau$, and $\varepsilon_{\text {trig }}\left(\gamma_{d_{1}}, \gamma_{d_{2}}\right)$ is the trigger efficiency given that the event is reconstructed. Eq. 3.10 assumes that the reconstruction probability for each of the lepton jets can be considered independently. Both $P_{\text {reco }}$ and $\varepsilon_{\text {trig }}$ depend on the event Type, and will be described presently.

The reconstruction efficiencies for a $\gamma_{d}$ with transverse momentum $p_{T}$ decaying at a length $L, \varepsilon_{\text {reco }} \equiv \varepsilon_{\text {reco }}\left(p_{T}, L\right)$, are provided in the ATLAS auxiliary tables $1-4$ [13] for Type 0 and Type 2 jets decaying in the barrel and endcap regions, as defined in table 1 . We assume $\varepsilon_{\text {reco }}=0$ outside of those $L, \eta, p_{T}$ regions, which appears to be stricter (and therefore more conservative) than the barrel/endcap regions used in the full analysis. Since the $\gamma_{d}$ are very boosted, we do not require a timing veto. The reconstruction probability for each jet is then

$$
P_{\text {reco }}\left(\gamma_{d}, c \tau\right)=\sum_{L \text { bins }} P\left(\gamma_{d}, c \tau\right) \varepsilon_{\text {reco }}\left(p_{T}^{\gamma_{d}}, L\right)
$$

where $P\left(\gamma_{d}, c \tau\right)$ is given by eq. (3.3) with

$$
\left(L_{\min }, L_{\max }\right)= \begin{cases}\left(\frac{L_{x y \text {-bin }}^{\min }}{\sin \theta}, \frac{L_{x y \text {-bin }}^{\max }}{\sin \theta}\right) & \text { if in barrel, } \\ \left(\frac{L_{z \text {-bin }}^{\min }}{\cos \theta}, \frac{L_{z \text {-bin }}^{\max }}{\cos \theta}\right) & \text { if in endcap. }\end{cases}
$$

For any event involving a Type 2 jet, ATLAS used the previously described CalRatio trigger. ATLAS provides the CalRatio trigger efficiency $\varepsilon_{\text {cal }}$, defined as the fraction of jets passing the offline selection which also pass the trigger, separately as a function of $p_{T}$ and $\eta$. Type 0-0 events are collected by the 3mu6_MSonly trigger $[12,13]$, which requires at least three standalone (not combined with an inner detector track) muons with $p_{T}>6 \mathrm{GeV}$.

\footnotetext{
${ }^{10}$ This term includes additional rejection criteria such as inner detector isolation.
} 


\begin{tabular}{|c|c|c|c|}
\hline \multicolumn{2}{|c|}{ Type 0 } & \multicolumn{2}{c|}{ Type 2} \\
\hline Barrel & Endcap & Barrel & Endcap \\
\hline $14 \leq L_{x y} / \mathrm{cm} \leq 780$ & $50 \leq L_{z} / \mathrm{cm} \leq 1400$ & $150 \leq L_{x y} / \mathrm{cm} \leq 410$ & $350 \leq L_{z} / \mathrm{cm} \leq 650$ \\
$|\eta|<0.9$ & $1.2<|\eta|<2.5$ & $|\eta|<1.0$ & $1.5<|\eta|<2.4$ \\
$10 \leq p_{T} / \mathrm{GeV} \leq 100$ & $10 \leq p_{T} / \mathrm{GeV} \leq 100$ & $20 \leq p_{T} / \mathrm{GeV} \leq 100$ & $20 \leq p_{T} / \mathrm{GeV} \leq 100$ \\
\hline
\end{tabular}

Table 1. Definitions of barrel and endcap regions for $\varepsilon_{\text {reco }}\left(p_{T}, L\right)$ as defined by ATLAS.

The efficiency of this trigger is dominated by the granularity of the muon spectrometer; to reconstruct three muons at least one of the dark photons must produce a pair of muons which have a discernible opening angle. ATLAS provides the efficiency $\varepsilon_{2}$, defined as the fraction of $\gamma_{d}$ passing the offline selection and also producing two distinguishable muons, separately as a function of $p_{T}$ and $\eta$. The efficiency for detecting at least one muon is quoted as $\varepsilon_{\geq 1}=0.8(0.9)$ in the barrel (endcap) region. For our purposes we converted these efficiencies, making the assumption of independence, into functions of two variables, $\varepsilon_{\text {cal }} \equiv \varepsilon_{\text {cal }}\left(p_{T}, \eta\right)$ and $\varepsilon_{2} \equiv \varepsilon_{2}\left(p_{T}, \eta\right)$. For each event, the trigger efficiency given event reconstruction is taken as

$$
\varepsilon_{\text {trig }}\left(\gamma_{d_{1}}, \gamma_{d_{2}}\right)= \begin{cases}\varepsilon_{\geq 1}\left(\gamma_{d 1}\right) \varepsilon_{2}\left(\gamma_{d 2}\right)+\varepsilon_{\geq 1}\left(\gamma_{d 2}\right) \varepsilon_{2}\left(\gamma_{d 1}\right)-\varepsilon_{2}\left(\gamma_{d 1}\right) \varepsilon_{2}\left(\gamma_{d 2}\right) & \text { if Type 0-0, } \\ \varepsilon_{\text {cal }}\left(\gamma_{d_{\text {Type- } 0}}\right) & \text { if Type 0-2, } \\ \varepsilon_{\text {cal }}\left(\gamma_{d_{1}}\right)+\varepsilon_{\text {cal }}\left(\gamma_{d_{2}}\right)-\varepsilon_{\text {cal }}\left(\gamma_{d_{1}}\right) \varepsilon_{\text {cal }}\left(\gamma_{d_{2}}\right) & \text { if Type 2-2, }\end{cases}
$$

where, in an obvious notation, $\varepsilon\left(\gamma_{d}\right) \equiv \varepsilon\left(p_{T}^{\gamma_{d}}, \eta^{\gamma_{d}}\right)$. This is not quite a model-independent trigger efficiency, since $\varepsilon_{\text {cal }}$ and $\varepsilon_{2}$ are derived from a lepton-jet gun event sample, in which the $\gamma_{d}$ are generated uniformly in $\left(p_{T}, \eta\right)$, but it serves as a good approximation for our purposes. After weighting by reconstruction probabilities, we find that this trigger efficiency for the FRVZ sample rescales the number of events by an approximately global number, $\approx 0.5$ for $c \tau_{\gamma_{d}}=0.1 \mathrm{~cm}$ and $\approx 0.3$ for $c \tau_{\gamma_{d}}=100 \mathrm{~cm}$.

In figure 2(a) we compare the number of events predicted by our analysis to those of ATLAS as a function of $c \tau$ assuming $100 \%$ Higgs branching. The obtained $\approx 330$ events at $c \tau=4.7 \mathrm{~cm}$ is an underprediction compared to the full simulation results of $600 \pm 40$, most likely due to the stricter barrel/endcap regions employed. For alternative lifetimes we cannot come up with a physical explanation that could account for the shape discrepancy between our curve and the reweighted result of ATLAS.

Now we wish to reinterpret the ATLAS analysis for $h \rightarrow \gamma_{d} \gamma_{d}$ decays predicted by the vector portal model described in section 2. A signal sample $g g \rightarrow h \rightarrow \gamma_{d} \gamma_{d}$ for $m_{\gamma_{d}}=400 \mathrm{MeV}$ was generated in PYTHIA and fed through our analysis. Figure 2(a) shows the total number of events predicted as a function of $c \tau$, as well as broken down by event Type. More events are predicted than in the FRVZ model, and they peak at a lower $c \tau$, since on average the $\gamma_{d}$ are more boosted. The $95 \%$ CL upper limit of $\approx 120(\approx 30)$ on the total (total excluding Type 2-2) number of events can be inferred from the ATLAS plots. 


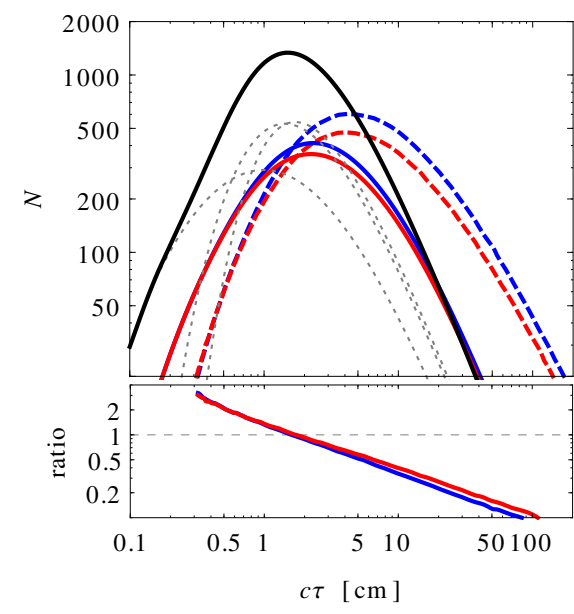

(a)

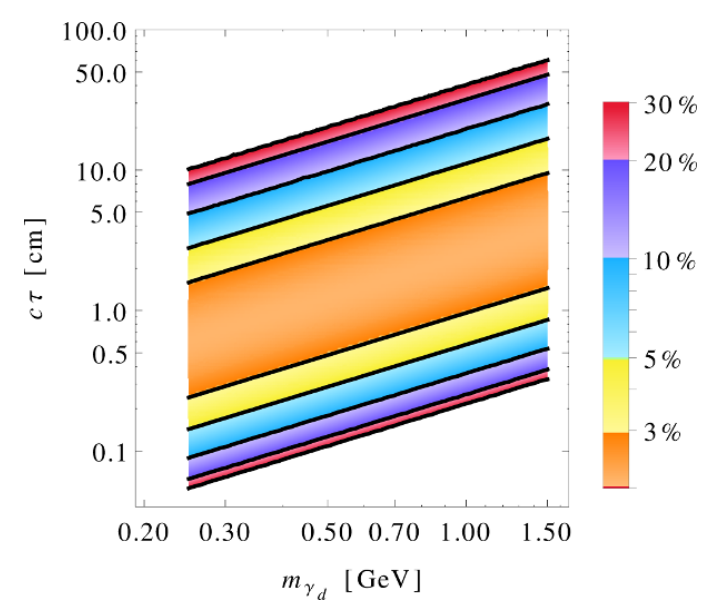

(b)

Figure 2. (a) Predicted number of events in the lepton jet analysis assuming $100 \%$ Higgs branching, $m_{\gamma_{d}}=400 \mathrm{MeV}$, and $\operatorname{Br}\left(\gamma_{d} \rightarrow\right.$ Type 0$)=0.45$. Upper: the FRVZ model results from ATLAS (dashed) and our results (solid) for all event Types (blue/upper) and excluding Type 2-2 events (red/lower). Also shown are the number of events expected for the $h \rightarrow \gamma_{d} \gamma_{d}$ model in solid black; dotted curves beginning left-to-right show the break down in terms of Type 0-0, 0-2, 2-2 events respectively. Lower: the ratio of our FRVZ model results to those of ATLAS. (b) Excluded parameter region for $\gamma_{d}$ assuming fixed $\operatorname{Br}\left(h \rightarrow \gamma_{d} \gamma_{d}\right) \times \operatorname{Br}\left(\gamma_{d} \rightarrow \mu \mu\right)^{2}$. The contours mark branchings of $30 \%, 20 \%, 10 \%, 5 \%, 3 \%$.

These numbers can be used along with figure 2(a) to limit exotic Higgs branching fractions for $m_{\gamma_{d}}=400 \mathrm{MeV}$.

For alternative masses, since $E \gg m$, the properties of the simulated $\gamma_{d}$ will be approximately the same but for the boost $\gamma=E / m_{\gamma_{d}}$. If the efficiencies do not change significantly surrounding $m_{\gamma_{d}}=400 \mathrm{MeV}$, which according to ATLAS is at least a good assumption for $0.25 \lesssim m_{\gamma_{d}} / \mathrm{GeV} \lesssim 1.5$ [13], then according to eq. (3.3) the number of events plotted as a function of $m_{\gamma_{d}} c \tau$ remains invariant. Limits for alternative masses can be derived from the $m_{\gamma_{d}}=400 \mathrm{MeV}$ results using this observation. In figure 2(b) we present an example exclusion plot derived from figure 2(a) in this way: the limit on $\operatorname{Br}\left(h \rightarrow \gamma_{d} \gamma_{d}\right) \times \operatorname{Br}\left(\gamma_{d} \rightarrow \mu \mu\right)^{2}$ as a function of $m_{\gamma_{d}}$ and $c \tau$. This is also a good approximation for the limit on $\operatorname{Br}(h \rightarrow s s) \times B r(s \rightarrow \mu \mu)^{2}$. In section 4, figure 2(b) (and related limits on branchings to the other event Types) are reinterpreted to bound mixing-mass parameter space for the Higgs and vector portals; there the exclusion is extended up to the $\tau \tau$ threshold $m_{\gamma_{d}} \approx 3.5 \mathrm{GeV}$.

\section{Portal limits}

In figures 3 and 4 we reinterpret the analyses of section 3 for the Higgs and vector portal models. The coloured regions mark the exclusion assuming $B r(h \rightarrow s s)=30 \%, 20 \%, 10 \%$, $5 \%, 3 \%$.

For the Higgs portal figure 3, we ask the reader to bear in mind that within the shaded region $2 m_{\pi}<m_{s} \lesssim 4 \mathrm{GeV}$ the branching fractions and lifetimes are known to be uncertain 


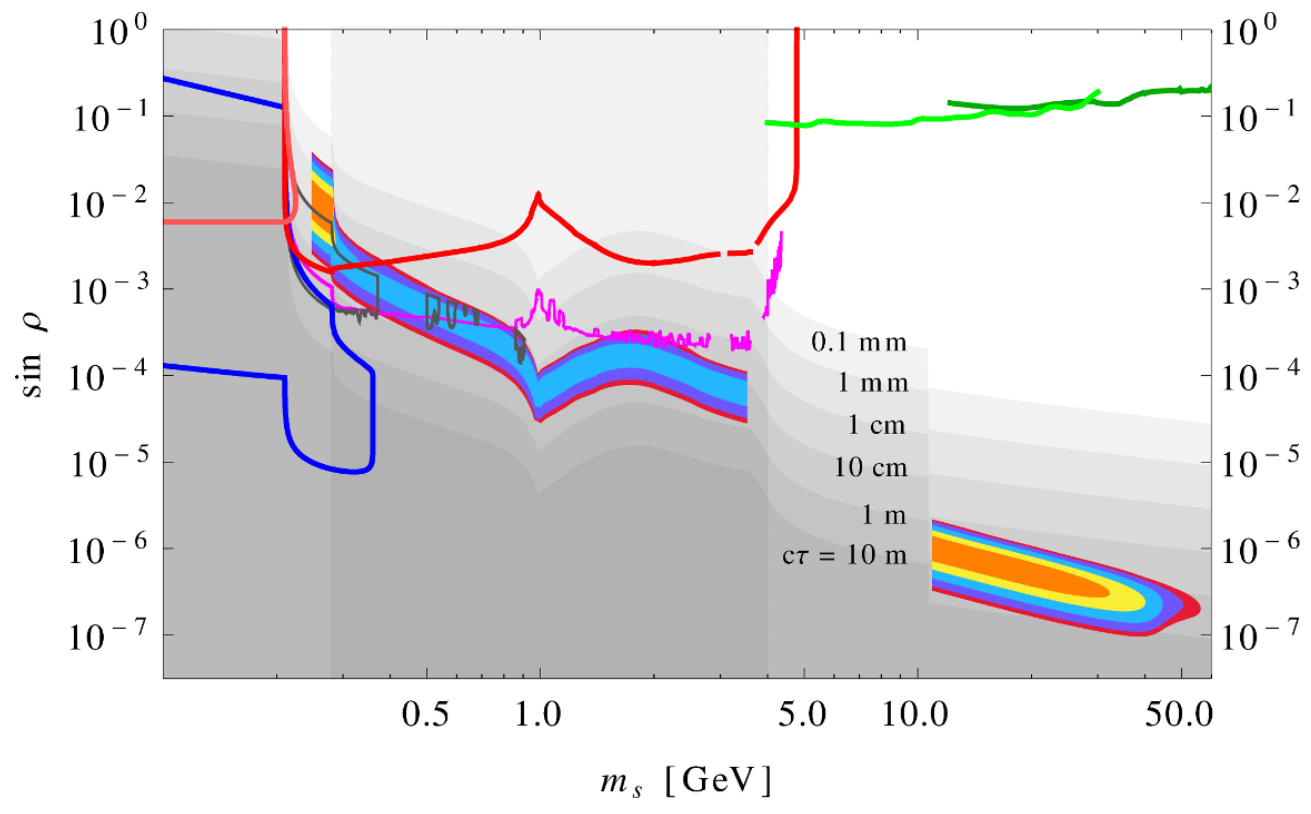

Figure 3. Exclusion plot for the real singlet scalar (Higgs) portal (see text). Incremental shadings mark areas of non-negligible lifetime. The coloured regions (this analysis) mark the exclusions assuming $\operatorname{Br}(h \rightarrow s s)=30 \%, 20 \%, 10 \%, 5 \%, 3 \%$.

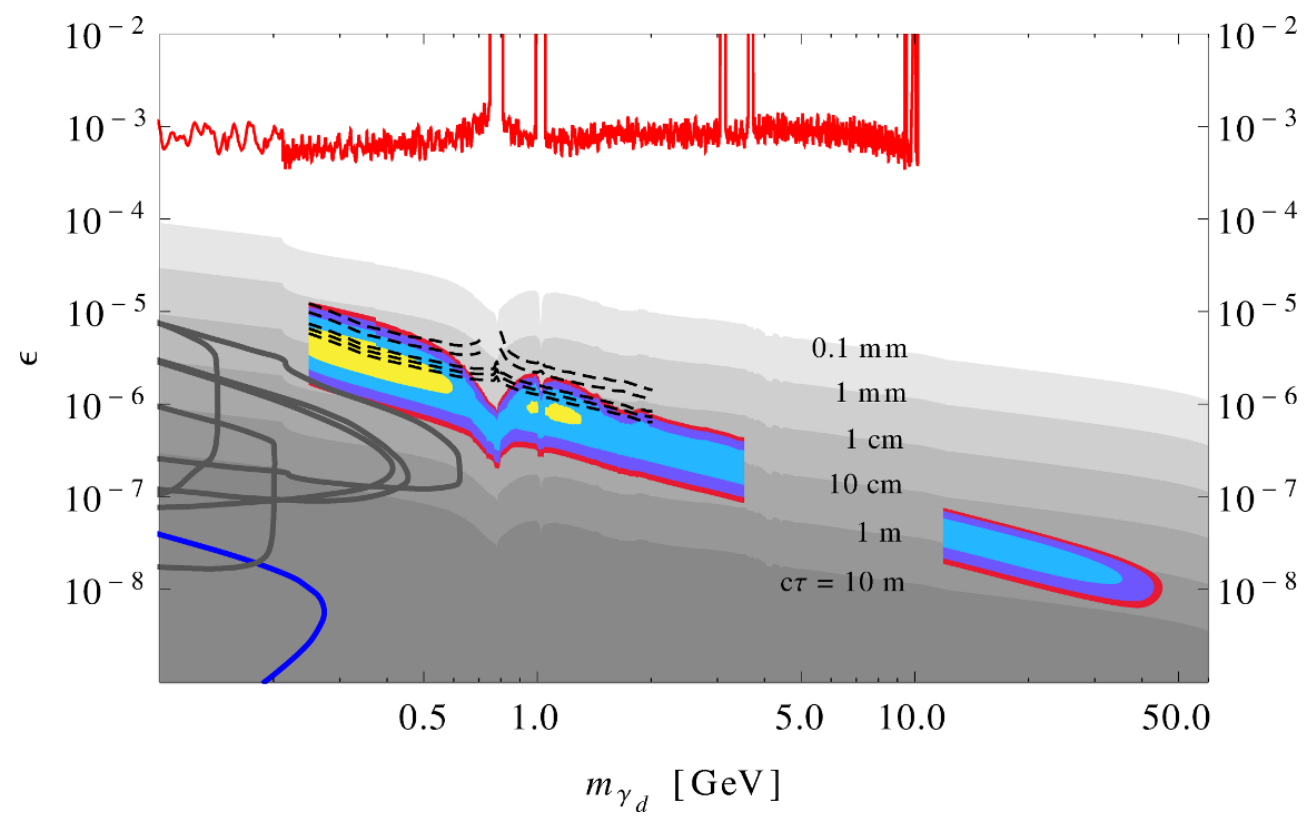

Figure 4. Exclusion plot for the vector portal (see text). Incremental shadings mark areas of non-negligible lifetime. The coloured regions (this analysis) and dashed lines (CMS ref. [64]) mark the exclusions assuming $\mathrm{Br}\left(h \rightarrow \gamma_{d} \gamma_{d}\right)=30 \%, 20 \%, 10 \%, 5 \%, 3 \%$. 
by up to an order of magnitude (see ref. [22]). We adopt the most recent calculation [65] below $1.4 \mathrm{GeV}$ and smoothly interpolate to a perturbative calculation [22] above $2 \mathrm{GeV}$. The limits that can be derived from figure 2(a) (such as in figure 2(b)) are independent of these uncertainties, so that there is enough information provided to reinterpret our results for alternative branching fractions and lifetimes; in all cases there is excluded parameter space with $\operatorname{Br}(h \rightarrow s s)<10 \%$. Limit lines (borrowed from ref. [22]) are from prompt $B$ decays at Belle/BaBar (reds) [66-69], the CHARM beam dump (blue) [70, 71], and LEP (greens) [72-74]. Note that prompt searches at LHCb will be more sensistive for $1 \lesssim$ $m_{s} / \mathrm{GeV} \lesssim 5$, but non-trivial long-lifetime effects must be carefully taken into account [75]. Very recently, LHCb presented a search [76] for long-lived light scalars in $B^{0} \rightarrow K^{* 0} s \rightarrow$ $\left(K^{ \pm} \pi^{\mp}\right)\left(\mu^{+} \mu^{-}\right)$decays; the limit shown in magenta is reinterpreted from their figure 4 assuming the branching expression found in ref. [77]. Also included are the excluded regions from a recent BaBar search for long-lived particles (dark grey) [78], reinterpreted from their figure $3\left(1 \mathrm{~cm} \leq c \tau_{s} \leq 100 \mathrm{~cm}\right)$ assuming $\operatorname{Br}\left(B \rightarrow s X_{s}\right) \approx 5.6 \sin ^{2} \rho\left(1-m_{s}^{2} / m_{B}^{2}\right)^{2}$ [79].

For the vector portal figure 4 , we calculated lifetimes and branchings for $m_{\gamma_{d}}<12 \mathrm{GeV}$ using the $R(s)=\sigma\left(e^{+} e^{-} \rightarrow\right.$ hadrons $) / \sigma\left(e^{+} e^{-} \rightarrow \mu^{+} \mu^{-}\right)$values collated by the PDG [80]. For $m_{\gamma_{d}} \geq 12 \mathrm{GeV}$ we used those values provided in ref. [36]. The exclusion lines are from a BaBar search (red) [81], supernovae cooling (blue) [82], and various beam dump experiments (dark grey) [83-87]. Limits from a recent CMS search [64] for prompt $h \rightarrow \gamma_{d} \gamma_{d}$ decays are reproduced as dashed lines assuming $\operatorname{Br}\left(h \rightarrow \gamma_{d} \gamma_{d}\right)=30 \%, 20 \%, 10 \%, 5 \%, 3 \%$, bottom-to-top.

\section{Conclusion}

Searching for the displaced decays of long-lived neutral states is a sensitive way to look for exotic Higgs physics. Already, searches at the LHC have probed branching fractions at the per cent level.

In section 2 we demonstrated how simple it is to build natural models with displaced Higgs decay phenomenology. Those models taken together serve to emphasise two points: (a) that long-lived neutral states can inherit a wide variety of couplings to SM particles, and (b) in extended models, many possible production mechanisms and final states exist. This motivates collaborations to present their results in as model-independent a way as is possible, and in section 3 we sought to explore by example how this might be done.

Unlike for prompt events, no public tool exists to simulate the detector response to displaced decays. Hence phenomenologists wanting to reinterpret displaced searches are reliant upon efficiencies provided by the collaborations. In general, the reconstruction efficiency for a long-lived particle will depend on its mass $m$, transverse momentum $p_{T}$, lab-frame decay length $L$, pseudorapidity $\eta$, and its decay mode. Ideally the phenomenologist would know the reconstruction efficiency as a function of all five of these parameters together, ${ }^{11}$ and then the simple Monte Carlo method we have described in section 3, requiring no simulation of displaced decays, could be used to determine the reconstruction

\footnotetext{
${ }^{11}$ Although it is only possible to provide a two-dimensional efficiency plot on paper, we see no reason why collaborations couldn't provide higher dimensional plots as an online resource.
} 
probability for any event. In the interests of simplicity, the dependence on $\eta$ is likely to be weak enough to be split into barrel and endcap regions, and then the reconstruction efficiencies could be provided as three-dimensional $\left(m, p_{T}, L\right)$ functions for each final state in the barrel/endcap. In certain limits the dependence on one of these variables might even be removed. For example, in the limit $E \gg m$, the efficiency dependence on $m$ for hadronic jets is expected to be weak. The trigger efficiency, defined as the probability of triggering given reconstruction, could subsequently be taken into account in a similar way.

Although this efficiency table wishlist was not fully realised for either of the ATLAS displaced searches considered in section 3 , we were still able to demonstrate the principles of a simple Monte Carlo method for reinterpretation, and we used it to constrain parameter space of interest for portal models (see also section 4). Our hope is that this paper inspires the following take-home message regarding displaced searches: if the relevant multidimensional efficiency tables are provided, then phenomenologists will be able to reinterpret searches in the context of their own models.

\section{Acknowledgments}

The author would like to thank Raymond Volkas and Robert Foot for invaluable support and advice, as well as James Barnard and Michael Schmidt for useful conversations. This work was supported in part by the Australian Research Council.

Open Access. This article is distributed under the terms of the Creative Commons Attribution License (CC-BY 4.0), which permits any use, distribution and reproduction in any medium, provided the original author(s) and source are credited.

\section{References}

[1] ATLAS collaboration, Observation of a new particle in the search for the standard model Higgs boson with the ATLAS detector at the LHC, Phys. Lett. B 716 (2013) 1 [arXiv: 1207.7214] [INSPIRE].

[2] CMS collaboration, Observation of a new boson at a mass of $125 \mathrm{GeV}$ with the CMS experiment at the LHC, Phys. Lett. B 716 (2013) 30 [arXiv:1207.7235] [INSPIRE].

[3] G. Bélanger, B. Dumont, U. Ellwanger, J.F. Gunion and S. Kraml, Global fit to Higgs signal strengths and couplings and implications for extended Higgs sectors, Phys. Rev. D 88 (2013) 075008 [arXiv: 1306.2941] [INSPIRE].

[4] K. Cheung, J.S. Lee and P.-Y. Tseng, Higgs precision analysis updates 2014, Phys. Rev. D 90 (2014) 095009 [arXiv: 1407.8236] [InSPIRE].

[5] M.J. Strassler and K.M. Zurek, Discovering the Higgs through highly-displaced vertices, Phys. Lett. B 661 (2008) 263 [hep-ph/0605193] [INSPIRE].

[6] R. Foot, A. Kobakhidze, K.L. McDonald and R.R. Volkas, Poincaré protection for a natural electroweak scale, Phys. Rev. D 89 (2014) 115018 [arXiv:1310.0223] [INSPIRE].

[7] N. Arkani-Hamed and N. Weiner, LHC signals for a superunified theory of dark matter, JHEP 12 (2008) 104 [arXiv:0810.0714] [inSPIRE]. 
[8] ATLAS collaboration, Search for a light Higgs boson decaying to long-lived weakly-interacting particles in proton-proton collisions at $\sqrt{s}=7$ TeV with the ATLAS detector, Phys. Rev. Lett. 108 (2012) 251801 [arXiv:1203.1303] [INSPIRE].

[9] ATLAS collaboration, Search for pair produced long-lived neutral particles decaying in the ATLAS hadronic calorimeter in pp collisions at $\sqrt{s}=8 \mathrm{TeV}$, ATLAS-CONF-2014-041 (2014).

[10] ATLAS collaboration, Search for pair-produced long-lived neutral particles decaying in the ATLAS hadronic calorimeter in pp collisions at $\sqrt{s}=8$ TeV, Phys. Lett. B 743 (2015) 15 [arXiv: 1501.04020] [INSPIRE].

[11] ATLAS collaboration, Search for long-lived, weakly interacting particles that decay to displaced hadronic jets in proton-proton collisions at $\sqrt{s}=8$ TeV with the ATLAS detector, Phys. Rev. D 92 (2015) 012010 [arXiv:1504.03634] [INSPIRE].

[12] ATLAS collaboration, Search for displaced muonic lepton jets from light Higgs boson decay in proton-proton collisions at $\sqrt{s}=7 \mathrm{TeV}$ with the ATLAS detector, Phys. Lett. B 721 (2013) 32 [arXiv: 1210.0435] [INSPIRE].

[13] ATLAS collaboration, Search for long-lived neutral particles decaying into lepton jets in proton-proton collisions at $\sqrt{s}=8 \mathrm{TeV}$ with the ATLAS detector, JHEP 11 (2014) 088 [arXiv: 1409.0746] [INSPIRE].

[14] CMS collaboration, Search in leptonic channels for heavy resonances decaying to long-lived neutral particles, JHEP 02 (2013) 085 [arXiv:1211.2472] [INSPIRE].

[15] CMS collaboration, Search for long-lived neutral particles decaying to quark-antiquark pairs in proton-proton collisions at $\sqrt{s}=8 \mathrm{TeV}$, Phys. Rev. D 91 (2015) 012007 [arXiv:1411.6530] [INSPIRE].

[16] CMS collaboration, Search for long-lived particles that decay into final states containing two electrons or two muons in proton-proton collisions at $\sqrt{s}=8 \mathrm{TeV}$, Phys. Rev. D 91 (2015) 052012 [arXiv: 1411.6977] [INSPIRE].

[17] LHCb collaboration, Search for Higgs-like bosons decaying into long-lived exotic particles, LHCb-CONF-2012-014 (2012).

[18] LHCb collaboration, Search for long-lived particles decaying to jet pairs, Eur. Phys. J. C 75 (2015) 152 [arXiv:1412.3021] [INSPIRE].

[19] V. Silveira and A. Zee, Scalar phantoms, Phys. Lett. B 161 (1985) 136 [inSPIRE].

[20] R. Foot, H. Lew and R.R. Volkas, A model with fundamental improper space-time symmetries, Phys. Lett. B 272 (1991) 67 [InSPIRE].

[21] B. Patt and F. Wilczek, Higgs-field portal into hidden sectors, hep-ph/0605188 [INSPIRE].

[22] J.D. Clarke, R. Foot and R.R. Volkas, Phenomenology of a very light scalar (100 MeV $<m_{h}<10 \mathrm{GeV}$ ) mixing with the SM Higgs, JHEP 02 (2014) 123 [arXiv:1310.8042] [INSPIRE].

[23] G.C. Branco, P.M. Ferreira, L. Lavoura, M.N. Rebelo, M. Sher and J.P. Silva, Theory and phenomenology of two-Higgs-doublet models, Phys. Rept. 516 (2012) 1 [arXiv:1106.0034] [INSPIRE].

[24] S.L. Glashow and S. Weinberg, Natural conservation laws for neutral currents, Phys. Rev. D 15 (1977) 1958 [INSPIRE]. 
[25] E.A. Paschos, Diagonal neutral currents, Phys. Rev. D 15 (1977) 1966 [inSPIRE].

[26] D. Curtin et al., Exotic decays of the 125 GeV Higgs boson, Phys. Rev. D 90 (2014) 075004 [arXiv: 1312.4992] [INSPIRE].

[27] B. Holdom, Two U(1)'s and epsilon charge shifts, Phys. Lett. B 166 (1986) 196 [InSPIRE].

[28] H. Davoudiasl, H.-S. Lee and W.J. Marciano, 'Dark' Z implications for parity violation, rare meson decays and Higgs physics, Phys. Rev. D 85 (2012) 115019 [arXiv:1203.2947] [INSPIRE].

[29] H.-S. Lee and M. Sher, Dark two Higgs doublet model, Phys. Rev. D 87 (2013) 115009 [arXiv: 1303.6653] [INSPIRE].

[30] F. Englert and R. Brout, Broken symmetry and the mass of gauge vector mesons, Phys. Rev. Lett. 13 (1964) 321 [INSPIRE].

[31] P.W. Higgs, Broken symmetries and the masses of gauge bosons, Phys. Rev. Lett. 13 (1964) 508 [INSPIRE].

[32] G.S. Guralnik, C.R. Hagen and T.W.B. Kibble, Global conservation laws and massless particles, Phys. Rev. Lett. 13 (1964) 585 [INSPIRE].

[33] S. Gopalakrishna, S. Jung and J.D. Wells, Higgs boson decays to four fermions through an abelian hidden sector, Phys. Rev. D 78 (2008) 055002 [arXiv:0801.3456] [INSPIRE].

[34] C.-F. Chang, E. Ma and T.-C. Yuan, Multilepton Higgs decays through the dark portal, JHEP 03 (2014) 054 [arXiv: 1308.6071] [INSPIRE].

[35] B. Batell, M. Pospelov and A. Ritz, Probing a secluded U(1) at B-factories, Phys. Rev. D 79 (2009) 115008 [arXiv:0903.0363] [INSPIRE].

[36] D. Curtin, R. Essig, S. Gori and J. Shelton, Illuminating dark photons with high-energy colliders, JHEP 02 (2015) 157 [arXiv: 1412.0018] [InSPIRE].

[37] M.L. Graesser, Broadening the Higgs boson with right-handed neutrinos and a higher dimension operator at the electroweak scale, Phys. Rev. D 76 (2007) 075006 [arXiv: 0704.0438] [INSPIRE].

[38] M.L. Graesser, Experimental constraints on Higgs boson decays to TeV-scale right-handed neutrinos, arXiv:0705.2190 [INSPIRE].

[39] D.G. Cerdeño, V. Martín-Lozano and O. Seto, Displaced vertices and long-lived charged particles in the NMSSM with right-handed sneutrinos, JHEP 05 (2014) 035 [arXiv:1311.7260] [INSPIRE].

[40] A. Maiezza, M. Nemevšek and F. Nesti, Lepton number violation in Higgs decay at LHC, Phys. Rev. Lett. 115 (2015) 081802 [arXiv:1503.06834] [INSPIRE].

[41] L.M. Carpenter, D.E. Kaplan and E.-J. Rhee, Reduced fine-tuning in supersymmetry with R-parity violation, Phys. Rev. Lett. 99 (2007) 211801 [hep-ph/0607204] [INSPIRE].

[42] D.E. Kaplan and K. Rehermann, Proposal for Higgs and superpartner searches at the LHCb experiment, JHEP 10 (2007) 056 [arXiv:0705.3426] [INSPIRE].

[43] D. Aristizabal Sierra, W. Porod, D. Restrepo and C.E. Yaguna, Novel Higgs decay signals in R-parity violating models, Phys. Rev. D 78 (2008) 015015 [arXiv:0804.1907] [INSPIRE].

[44] P.W. Graham, D.E. Kaplan, S. Rajendran and P. Saraswat, Displaced supersymmetry, JHEP 07 (2012) 149 [arXiv: 1204.6038] [INSPIRE]. 
[45] P. Ghosh, D.E. Lopez-Fogliani, V.A. Mitsou, C. Muñoz and R.R. de Austri, Probing the $\mu \nu S S M$ with light scalars, pseudoscalars and neutralinos from the decay of a SM-like Higgs boson at the LHC, JHEP 11 (2014) 102 [arXiv:1410.2070] [INSPIRE].

[46] Y. Cui and B. Shuve, Probing baryogenesis with displaced vertices at the LHC, JHEP 02 (2015) 049 [arXiv: 1409.6729] [INSPIRE].

[47] Y.F. Chan, M. Low, D.E. Morrissey and A.P. Spray, LHC signatures of a minimal supersymmetric hidden valley, JHEP 05 (2012) 155 [arXiv:1112.2705] [INSPIRE].

[48] A. Falkowski, J.T. Ruderman, T. Volansky and J. Zupan, Hidden Higgs decaying to lepton jets, JHEP 05 (2010) 077 [arXiv: 1002.2952] [INSPIRE].

[49] A. Falkowski, J.T. Ruderman, T. Volansky and J. Zupan, Discovering Higgs decays to lepton jets at hadron colliders, Phys. Rev. Lett. 105 (2010) 241801 [arXiv:1007.3496] [INSPIRE].

[50] M.J. Strassler and K.M. Zurek, Echoes of a hidden valley at hadron colliders, Phys. Lett. B 651 (2007) 374 [hep-ph/0604261] [INSPIRE].

[51] J.E. Juknevich, Pure-glue hidden valleys through the Higgs portal, JHEP 08 (2010) 121 [arXiv:0911.5616] [INSPIRE].

[52] N. Craig, A. Katz, M. Strassler and R. Sundrum, Naturalness in the Dark at the LHC, JHEP 07 (2015) 105 [arXiv: 1501.05310] [INSPIRE].

[53] D. Curtin and C.B. Verhaaren, Discovering uncolored naturalness in exotic Higgs decays, arXiv: 1506.06141 [INSPIRE].

[54] C. Csáki, E. Kuflik, S. Lombardo and O. Slone, Searching for displaced Higgs decays, arXiv: 1508.01522 [INSPIRE].

[55] M.J. Strassler, Possible effects of a hidden valley on supersymmetric phenomenology, hep-ph/0607160 [INSPIRE].

[56] T. Han, Z. Si, K.M. Zurek and M.J. Strassler, Phenomenology of hidden valleys at hadron colliders, JHEP 07 (2008) 008 [arXiv:0712.2041] [INSPIRE].

[57] P. Schwaller, D. Stolarski and A. Weiler, Emerging jets, JHEP 05 (2015) 059 [arXiv: 1502.05409] [INSPIRE].

[58] T. Sjöstrand, S. Mrenna and P.Z. Skands, PYTHIA 6.4 physics and manual, JHEP 05 (2006) 026 [hep-ph/0603175] [INSPIRE].

[59] T. Sjöstrand, S. Mrenna and P.Z. Skands, A brief introduction to PYTHIA 8.1, Comput. Phys. Commun. 178 (2008) 852 [arXiv:0710.3820] [InSPIRE].

[60] E. Conte, B. Fuks and G. Serret, MadAnalysis 5, a user-friendly framework for collider phenomenology, Comput. Phys. Commun. 184 (2013) 222 [arXiv:1206.1599] [INSPIRE].

[61] Z. Liu and B. Tweedie, The fate of long-lived superparticles with hadronic decays after LHC Run 1, JHEP 06 (2015) 042 [arXiv: 1503.05923] [InSPIRE].

[62] ATLAS collaboration, Triggering on long-lived neutral particles in the ATLAS detector, 2013 JINST 8 P07015 [arXiv:1305.2284].

[63] ATLAS collaboration, Triggers for displaced decays of long-lived neutral particles in the ATLAS detector, 2013 JINST 8 P07015 [arXiv: 1305.2284] [INSPIRE].

[64] CMS collaboration, A search for pair production of new light bosons decaying into muons, arXiv: 1506.00424 [INSPIRE]. 
[65] J.F. Donoghue, J. Gasser and H. Leutwyler, The decay of a light Higgs boson, Nucl. Phys. B 343 (1990) 341 [INSPIRE].

[66] Belle collaboration, K.F. Chen et al., Search for $B \rightarrow h^{*} \nu \overline{n u}$ decays at Belle, Phys. Rev. Lett. 99 (2007) 221802 [arXiv:0707.0138] [INSPIRE].

[67] BaBar collaboration, P. del Amo Sanchez et al., Search for the rare decay $B \rightarrow K \nu \overline{n u}$, Phys. Rev. D 82 (2010) 112002 [arXiv:1009.1529] [InSPIRE].

[68] Belle collaboration, J.T. Wei et al., Measurement of the differential branching fraction and forward-backword asymmetry for $B \rightarrow K^{(*)} \ell^{+} \ell^{-}$, Phys. Rev. Lett. 103 (2009) 171801 [arXiv: 0904.0770] [INSPIRE].

[69] BABAR collaboration, B. Aubert et al., Direct CP, lepton flavor and isospin asymmetries in the decays $B \rightarrow K^{(*)} \ell^{+} \ell^{-}$, Phys. Rev. Lett. 102 (2009) 091803 [arXiv:0807.4119] [inSPIRE].

[70] CHARM collaboration, F. Bergsma et al., Search for axion like particle production in $400 \mathrm{GeV}$ proton-copper interactions, Phys. Lett. B 157 (1985) 458 [INSPIRE].

[71] F. Bezrukov and D. Gorbunov, Light inflaton hunter's guide, JHEP 05 (2010) 010 [arXiv: 0912.0390] [INSPIRE].

[72] ALEPH collaboration, D. Buskulic et al., Search for a nonminimal Higgs boson produced in the reaction $e^{+} e^{-} \rightarrow h Z^{*}$, Phys. Lett. B 313 (1993) 312 [INSPIRE].

[73] L3 collaboration, M. Acciarri et al., Search for neutral Higgs boson production through the process $e^{+} e^{-} \rightarrow Z^{*} H^{0}$, Phys. Lett. B 385 (1996) 454 [INSPIRE].

[74] OPAL, DELPHI, LEP Working Group for Higgs boson searches, ALEPH, L3 collaboration, R. Barate et al., Search for the standard model Higgs boson at LEP, Phys. Lett. B 565 (2003) 61 [hep-ex/0306033] [INSPIRE].

[75] K. Schmidt-Hoberg, F. Staub and M.W. Winkler, Constraints on light mediators: confronting dark matter searches with B physics, Phys. Lett. B 727 (2013) 506 [arXiv: 1310.6752] [INSPIRE].

[76] LHCb collaboration, Search for hidden-sector bosons in $B^{0} \rightarrow K^{* 0} \mu^{+} \mu^{-}$decays, arXiv: 1508.04094 [INSPIRE].

[77] B. Batell, M. Pospelov and A. Ritz, Multi-lepton signatures of a hidden sector in rare B decays, Phys. Rev. D 83 (2011) 054005 [arXiv:0911.4938] [INSPIRE].

[78] BaBAR collaboration, J.P. Lees et al., Search for long-lived particles in $e^{+} e^{-}$collisions, Phys. Rev. Lett. 114 (2015) 171801 [arXiv:1502.02580] [INSPIRE].

[79] B. Grinstein, L.J. Hall and L. Randall, Do B meson decays exclude a light Higgs?, Phys. Lett. B 211 (1988) 363 [INSPIRE].

[80] Particle Data Group collaboration, K.A. Olive et al., Review of particle physics, Chin. Phys. C 38 (2014) 090001 [inSPIRE].

[81] BABAR collaboration, J.P. Lees et al., Search for a dark photon in $e^{+} e^{-}$collisions at BaBar, Phys. Rev. Lett. 113 (2014) 201801 [arXiv: 1406.2980] [INSPIRE].

[82] J.B. Dent, F. Ferrer and L.M. Krauss, Constraints on light hidden sector gauge bosons from supernova cooling, arXiv:1201.2683 [INSPIRE].

[83] J. Blumlein and J. Brunner, New exclusion limits for dark gauge forces from beam-dump data, Phys. Lett. B 701 (2011) 155 [arXiv:1104.2747] [INSPIRE]. 
[84] J. Blümlein and J. Brunner, New exclusion limits on dark gauge forces from proton bremsstrahlung in beam-dump data, Phys. Lett. B 731 (2014) 320 [arXiv:1311.3870] [INSPIRE].

[85] S.N. Gninenko, Constraints on sub-GeV hidden sector gauge bosons from a search for heavy neutrino decays, Phys. Lett. B 713 (2012) 244 [arXiv:1204.3583] [INSPIRE].

[86] J.D. Bjorken, R. Essig, P. Schuster and N. Toro, New fixed-target experiments to search for dark gauge forces, Phys. Rev. D 80 (2009) 075018 [arXiv: 0906.0580] [INSPIRE].

[87] R. Essig, R. Harnik, J. Kaplan and N. Toro, Discovering new light states at neutrino experiments, Phys. Rev. D 82 (2010) 113008 [arXiv:1008.0636] [InSPIRE]. 